
$\checkmark$ Research Square
Preprints are preliminary reports that have not undergone peer review.
They should not be considered conclusive, used to inform clinical practice, or referenced by the media as validated information.

\title{
How community engagement in water and sanitation interventions impacts human health, infrastructure and service longevity in low- and middle-income countries: a realist review
}

\author{
Sarah Nelson ( $\nabla$ s.a.nelson@live.com ) \\ The University of Sydney \\ Dorothy Drabarek \\ The University of Sydney \\ Aaron Jenkins \\ The University of Sydney \\ Joel Negin
}

The University of Sydney

Seye Abimbola

The University of Sydney

\section{Research article}

Keywords: Context-mechanism-outcome configuration, low- and middle-income countries, water, realist review, health, WASH, sustainability, communities, sanitation

Posted Date: April 10th, 2020

DOI: https://doi.org/10.21203/rs.3.rs-20891/v1

License: (c) (i) This work is licensed under a Creative Commons Attribution 4.0 International License.

Read Full License 


\section{Abstract}

Background Access to safe water and sanitation is considered a basic human right and a focus of the Sustainable Development Goals. However, many water and sanitation interventions do not have long lasting impacts in communities and are generally associated with high levels of failure. Limited information is available to understand what types of interventions work for whom and under what circumstances.

Methods A realist review to understand how, and under what circumstances community engagement in water and sanitation interventions impacts access (to safe water and sanitation), change (in health status or behaviour), and sustainability (of resources and services). We systematically searched PubMed, Web of Science and Scopus databases to identify studies from low- and middle-income countries.

Results We reviewed 56 studies conducted in 25 countries. We identified five mechanisms that explain access, change and sustainability outcomes: 1 ) accountability (policies and procedures to hold communities and committees responsible for their actions and outcomes of an initiative), 2) diffusion (spread of an idea or behaviour by innovators over time through communication amongst members of a community), 3) market (the interplay between demand and supply of a service or resource), 4) ownership (sense of possession and control of the service or resource), and 5) shame (a feeling of disgust in one's behaviour or actions). Contextual elements identified include: community location, communities with similar characteristics, communication, leadership, fines/penalties, seasonality, resource dependency, access to resources and funding, community financial and technical skills and knowledge, leadership, ongoing support and acknowledgement of change, community connectedness and social cohesion, community willingness to pay, committees that followed and understood responsibilities, rules and management plans, active committees with women and community/committee involvement in the design, planning or implementation of the intervention.

Conclusion The findings highlight five key mechanisms, impacted by 19 contextual factors that explain outcomes of community-based water and sanitation interventions. Policy makers, program implementers and institutions need to consider community dynamics, location, resources, committees and level of community involvement, prior to the introduction of community-based water and sanitation interventions.

\section{Background}

Access to safe water and sanitation is fundamental for human health (1). Water, sanitation and hygiene (WASH) interventions and initiatives continue to be the cornerstone of improving access to and the safety of these services, especially in low- and middle-income countries (LMICs). However, the literature reveals mixed results of these interventions and initiatives - some display positive impacts, with others showing no impact on health $(2,3)$. There is an expanding array of literature seeking to understand the contextual of WASH interventions and initiatives and their influence (4-7). It is important to understand the 
contextual factors that influence the success (or failure) of community based WASH interventions and initiatives, as no single strategy will be successful in all contexts and situations $(1,8,9)$.

WASH interventions are broad and rely on a broad range of factors such as cultural traditions $(10,11)$, resource dependency (12), quality and satisfaction (3), and community rules and procedures (13). Worldwide, more than a quarter of rural water infrastructure fails to function as intended (9), with around $70 \%$ in Sub Saharan African countries classified as non-functional (14). The sustainability required for resources and interventions to be maintained is often unrealistic, leading to failure (1). It is difficult for cities, especially in LMICs, to improve sanitation and expand potable water access while keeping up with population growth and migration (2).

Failure of interventions can occur for several reasons, such as lack of community involvement in design $(15,16)$, lack of ownership $(17,18)$, abuse of funds or poor financial management, lack of willingness of community members to contribute (18-20), lack of communication and connectedness (21) and no ongoing support and acknowledgement of change (22-24). In addition to an increased understanding of success and failures in WASH interventions, there is a growing trend to use community participation to drive health programmes, based on the expectation working with communities can help ensure interventions are more relevant to local priorities (25).

Current literature shows multiple benefits of community engagement. Community engagement allows cultural exchange and the building of knowledge between the implementing partners (7). In addition, communities, (particularly indigenous communities), have developed knowledge structures by place, space and relationality over generations that are passed from one generation to the next, providing information on how to use resources sustainably (7). Limited literature is available on collaborative action in health interventions in LMICs, partly due to the difficulty to implement it (26). Further, lack of community participation is often seen as a hindrance in collaborative action (27).

The definition and manifestation of community participation in WASH initiatives vary significantly across articles and studies (4). However, understanding the impact of contextual factors is important for designing and implementing long lasting interventions and initiatives within communities, given the large amount of heterogeneity within communities and interventions (6). Some reviews have been conducted to understand the impact of context on water and sanitation interventions, but none have attempted to synthesise the effects of multiple interacting contextual factors from the range of interventions. Rather, these reviews have tended to be realist reviews focusing on one contextual factor or a single intervention $(4,6)$.

Our realist review attempts to address this gap in the literature. The objective of the review was to identify mechanisms through which community engagement in water and sanitation interventions impact health, water, sanitation and sustainability outcomes in different circumstances or contexts in LMICs. Currently health and sustainability are addressed separately in the literature, but it is important to consider both together as they can exist in tension with one another as factors beneficial for human health do not 
always reflect the need for resources. Realist reviews generates an understanding of why a mechanism works in one setting and not in another (28).

This realist review was conducted to understand how and under what circumstances community engagement in water and sanitation interventions impacts access (to safe water and sanitation), change (in health status or behaviour), and sustainability (of these resources and services).

\section{Methods}

The review followed realist review guidelines as it allows evaluation of complex social interventions that cannot be examined by a systematic review process $(28,29)$. The realist approach begins with the premise that interventions are complex, and changes are influenced by and dependent on context (28). An outcome is determined by mechanisms offered or triggered by an intervention, which is in turn influenced by the context in which the intervention occurs in (28). The Context-Mechanism-Outcome (CMO) configurations demonstrate how they influence and shape one another. Different interventions can have different outcomes due to the contextual factors altering the mechanisms (28).

\section{Step 1: Development of review question}

The scope of the review was to answer how (e.g. community led initiatives or government led) and under what circumstances (e.g. location, community demographics, leadership) community engagement in water and sanitation interventions impacts access, change in health and behaviour, and sustainability. Initial scoping exercises identified individual contextual factors e.g. leadership $(30,31)$ and monitoring $(32,33)$, and in some case mechanisms for individual types of WASH interventions e.g. community ledtotal sanitation $(34,35)$. However, nothing was found to explain different types of outcomes, across multiple mechanisms.

\section{Step 2: Search strategy}

A literature search of PubMed, Web of Science and Scopus was conducted to find peer-reviewed journal articles that studied how community engagement in water and sanitation interventions impact health, water, sanitation and sustainability outcomes in LMICs. The search terms used were 'water', 'WASH', 'water resource', 'hygiene', 'sanitation', 'community participation', 'demand driven', 'community led', 'community engage*', 'community based', 'community manage', 'sustain ${ }^{\star \prime}$ and 'health'. The search words were adapted to the requirement of each database. Filters were applied to exclude reviews. Only articles from 01-01-2010 to 03-04-2019 were considered in the search. Only English articles were considered. Articles were identified and exported into Zotero. Duplicates were identified and removed.

\section{Step 3: Screening and study selection}

Initially, titles were screened independently by 2 reviewers. Discrepancies were discussed and resolved, and if there was no resolution, a third reviewer made the final decision on inclusion. 
In the second phase, abstracts were assessed to determine whether the study had a community role, was in a LMIC and that there was a link with health or sustainability. Abstracts were independently screened by two reviewers. Discrepancies were discussed and resolved, and if no resolution, the third reviewer made the final decision on inclusion. Finally, the third reviewer reviewed the 'yes' list of included abstracts, and discrepancies were then discussed amongst the three reviewers.

\section{Inclusion criteria}

After screening the abstract, full papers were assessed for inclusion if they met the following criteria:

\section{Initiative}

A new intervention or organised activity in the community with the intention to improve water quality; water access; sanitation; sustainability of water supply for human (and agricultural) use; environmental sustainability (water) and health. The intervention or organised activity could be endogenous or exogenous in origin.

\section{Outcome of interest}

The intervention or organised activity had an intended outcome linked to water, sanitation, health or sustainability.

\section{Community role}

The community had to participate or engage in one or more of the following ways

1.

Community had full authority in decision-making and autonomy of the management of water resource or initiative.

2.

Community had majority of authority in decision-making and/or management of water resource or initiative, whether the latter is endogenous or exogenous in origin.

3.

Community members (e.g. leaders, community health workers) were involved in the design and/or delivery of an initiative by an external agent. Community members may have varying degrees of autonomy in their work and decision-making power.

4.

Community had a role through participation, consultation or engagement in activities and actions of an initiative by an external agent. Levels of decision-making power of community members may vary and may be as low as being easily overruled by an external agent.

\section{Low- and middle-income countries}


Studies conducted in LMIC countries based on World Bank definitions (36).

\section{Study/Paper type}

Studies needed to include primary data.

\section{Language}

The full article needed to be available in English.

\section{Exclusion criteria:}

Papers were excluded based on the following criteria

(1)

Contained secondary data only, or was a review or a commentary

(2)

It discussed existing management practice for water and health resources

(3)

The outcome was not linked to linked to water, sanitation, health or sustainability

(4)

There was no clear community role in the intervention or organised activity

(5)

The study country was from a high-income country based, as defined by the World Bank (36)

(6)

The paper contained secondary data, or was a review and a commentary piece

(7)

The paper was not available in English.

\section{Step 4: Data extraction}

Five articles were randomly selected, and iterative data extraction was conducted independently by three of the authors. Data from the articles were extracted into an excel spreadsheet summarising the study population, country, community role, intervention, sanitation or water linkage, study type, context, mechanism and outcome components. Each article was examined for 'outcomes' linked to access to resources or services, behaviour change, changes in health status and sustainability of the resource or service. We identified factors (socioeconomic, geographical and institutional) that led to success or failure in relation to the outcomes, and these were categorised as context. The three researchers compared and discussed the findings to ensure they reached consensus in understanding. The remaining articles were divided between two researchers. Weekly meetings were held to discuss the data extraction and findings. Discrepancies were discussed and resolved among the research team.

\section{Step 5: Data analysis and synthesis}


After data was extracted, CMO relationships were mapped, identified and discussed amongst the research team. The outcomes were used as starting point for the analysis and this allowed for multiple mechanisms to be identified in each paper (37). The mechanisms were identified using both inductive and deductive reasoning to explain the context-outcome relationships. Patterns in CMOs were identified from the different articles and were used to build detailed CMO configurations. We then sought to better understand and define the identified mechanisms by exploring the wider literature in the field. We identified five mechanisms based on the evidence and patterns of CMO configurations in the information available in the included studies.

\section{Results}

The searches conducted in April 2019 identified 3,054 articles. Figure 1 outlines the study selection process. Overall, 56 papers were identified from 25 countries. By synthesising the information in each paper, we identified five mechanisms that explain the outcomes from the studies: accountability, diffusion, market, ownership and shame. Articles had multiple mechanisms. Across the five mechanisms three sets of outcomes were identified:

1. Access (to resources and services such as latrines, clean water, and sanitation) at individual or community level,

2. Change (to prevent disease, such as reduction in open defection and increase use of handwashing, or reduction in disease levels, or change in health status such as diarrheal rates) at individual or community level,

3. Sustainability (of water and sanitation resources or services, including factors that impact on their long-term sustainability, such as technical capacity to repair, operate and maintain a resource or service).

The next section describes each mechanism in detail, followed by a description of each based on individual outcome, and key contextual factors highlighted as identified through the literature review. See Table 1 for a summary.

\section{ACCOUNTABILITY}

Accountability is a formal mechanism triggered by rules, procedures and policies to hold committees, communities and households responsible for decisions and actions. It can help ensure that actions and decisions taken are appropriate to promote access, behaviour change and resource sustainability. The three mechanisms identified were access, change and sustainability. 


\section{Access}

Triggered by internal or external monitoring, accountability may result in improved community access to a resource or service. The focus of such monitoring may be progress towards achieving a goal. The goal may be about access to a service - e.g., to ensure that sanitation facilities are in place $(10,22,31,33)$, that facilities meet the needs of people with disabilities $(38)$, or that water is accessible $(39,40)$. The goal may also be resource preservation (safety and/or accessibility) so that water is available to be accessed as desired - e.g., to check water samples for faecal or arsenic contamination $(15,41)$, or to assess the level of groundwater available for agriculture throughout the year (32). Internal monitoring can be conducted by a chief or village headman $(10,22,31)$, locally trained volunteers $(41)$, community members (32) or by the community's health or water committee (39-41). External monitoring can be conducted by NGO facilitators $(38)$ or health workers $(33,42)$.

Opportunities and platforms for regular communication and meetings between community members, service users and health or water committees helps build a sense of trust and connection between all parties and helps to hold them accountable $(38,41)$. This is reinforced where community leadership is transparent and has open channels for communicating with community members $(10,22,31,40)$ and the use of technology allows up to date monitoring (31). Accountability is also strengthened where there is a functional, long-standing water or health committee whose members are active, motivated and committed, and have clear roles and responsibilities $(39,40)$. A gender balanced committee opens up the space for accountability, as more opinions and views are considered on what factors are important and need to be considered $(39,40,43,44)$.

\section{Change}

Ongoing external monitoring can hold communities accountable for changing their health and sanitation behaviours $(35,38,42,45)$, leading to improved health of children as seen in height and weight changes and diarrhoea occurrence (35). Without ongoing external monitoring, loss of momentum for change can occur, halting behaviour change progress (23). Ongoing monitoring is enabled by regular meetings and sharing of information to enforce behaviour change $(25,41)$. However, limited access to communities due to remote location, violence and large community size and layout are barriers to ongoing external monitoring $(35,40)$. Accountability can also influence behaviour change, when triggered by fines (e.g. money, goat or chicken) imposed as penalty for not conforming to a desired behaviour. For example, penalties can be used to enforce standards for latrines (25), for not constructing a latrine, and for open defection (10). Trust is an essential contextual factor for such penalties - tensions arise when a community does not trust the person or committee collecting the fines and what they will do with the fines $(18,25)$. Trust depends on the person or committee's record of financial management, and whether they have clear rules about the use of such penalties. 


\section{Sustainability}

Accountability within community committees can facilitate their continued activities, therefore promoting the sustainability of resources and services for which they are responsible. This requires that committees have internal feedback systems, take minutes and set agendas with written working plans, reflecting high self-organising capabilities, and a sense of obligation among committee members to attend meetings and take action to manage and maintain resources, thus contributing to sustainability of water and sanitation initiatives $(17,25,39,41,46,47)$. Accountability to government entities (e.g., water boards) for reports and abiding by government policies can promote sustainability as it can hold committees and communities responsible for funds to maintain resources and services $(15,17,48,49)$. Communication with the broader community holds committees accountable for their roles and responsibilities in relation to water and sanitation infrastructure in a community $(17,49,50)$. Communication can help enable the diffusion of efforts to change the landscape of governance within local communities and check progress of actions $(10,20)$.

Committee accountability is enabled when committees have clear roles, responsibilities, rules and procedures (which they understand) on responding to system breakdowns, and managing ongoing maintenance and missing parts, with direct implications for the sustainability of water access and WASH infrastructure $(21,25,39)$. Sustainability of resources and services is facilitated by a committee's ability to make funds available for operational and maintenance costs, and by it having rules and procedures for funds management (e.g., having a well-educated treasurer who manages and collects funds within the community), or by having the community participate actively in monitoring committee finances $(15,19,39,47)$. However, where there are known instances of abuse of funds or poor financial management, community members are less willing to contribute, leading to low sustainability of resources or services (18-20). Committee activity and social participation play key roles in managing maintenance and operation of water and sanitation systems and assist in management of funds ensuring continued service provision $(16,43,51,52)$. This can promote good governance and practice (16). While committee activity and social participation is an enabling factor, this alone is not enough to ensure the sustainability of community water and sanitation infrastructure (53).

\section{DIFFUSION}

Diffusion reflects the spread of an idea or behaviour by "innovators" (i.e. individuals who are the first to develop and try out new ideas) and "early adopters" (i.e. opinion leaders who enjoy leadership roles, and embrace change opportunities) over time through their influence and through communication amongst members of a community $(34,54)$. They spread innovations by using their shared knowledge of the 
language, social, cultural and religious beliefs and their existing relationships with and within communities $(30,31)$. Innovators and early adopters in a community include teachers $(30,55)$, leaders $(10,22,30,31,34,55,56)$, healthcare workers $(30,42,55,57,58)$, and community committees $(21,46,59,60)$. Diffusion can contribute to the three outcomes - access, change and sustainability.

\section{Access}

Access to resources such as latrines $(8,11,31,46,56,61,62)$, water $(33,50,60,63,64)$ and handwashing facilities (62) can be driven by diffusion. When these facilities or the idea of them are new (i.e. when they are "innovations" in a community), the collective action necessary to build and maintain them depends on the extent to which appreciation for their value spreads in the community. Information on communityled initiatives, and on initiatives that reflect the needs of the community take root more readily than initiatives driven from external sources. Information about the need of the resource or service can diffuse more readily where people have similar characteristics such as occupations and religion, have high levels of social connectedness and social cohesion and respect for one another. This means people are more concerned about others, are more likely to cooperate, communicate and work with one another, and agree on community priorities. Thus, it helps to promote change in resource access, as through information spreads by 'early adopters' and in the community helps to promote change in resource access as other community members are able to see the benefits $(8,46,61)$.

In such communities, innovators draw on the existing influence of leaders to set new social norms $(11,31,56)$, and early adopters draw on the existing skills within the community to work on a resource or intervention $(31,46,50,60)$. However, to have an impact on access within a community, diffusion does not need to occur across the whole community, it may just occur within sections of the community such as among women and other community groups $(11,60)$.

\section{Change}

Diffusion can occur through communication among peers, which leads to shared understanding, therefore reinforcing the adoption of new behaviour at an individual or community level $(30,31,46,63)$. Examples of diffusion influencing behaviour change include reduction of open defecation $(10,34,45,48,55,65)$, water purification (63), disposable of rubbish and care of animals (45), and sanitation and hygiene behaviours (60), which may lead to improved health outcomes such as reduction in diarrhoea rates and waterborne diseases $(42,60)$. Behaviour change is also further enabled by diffusion in communities with high levels of social cohesion and connectedness - by enabling communication among peers, to build and share knowledge $(46,62)$, and as highly regarded leaders influence the spread of new behaviour and create new social norms through their endorsement $(11,20,34,55)$. Higher levels of 
social cohesion and connectedness in small rural and remote communities can also help sustain longterm behaviour change (e.g., reduction in open defecation) especially where people stick together to create a supportive environment for long lasting change. In these contexts the focus is on behaviour change at the community rather than individual level $(45,55)$.

\section{Sustainability}

The sustainability of a water or sanitation resource or service may depend on the extent to which knowledge of its value diffuse and take root in the community, leading to greater understanding of the potential impact of sustaining the resource or service. The level of social cohesion and connectedness in a community in the form of shared norms, trust, a common sense of identity help facilitate spread information on new ideas $(30,46,50,56)$. The perceived need to collectively mobilise resources to address water or sanitation problems is shared by committees and within committees $(50,59,63,66,67)$. Willingness of community members to help one another further allows the diffusion to lead to sustainability of resources such as latrines $(8,62)$ and water access $(59,63)$, through the maintenance of the resources (50). Gender-balanced committees, or the active involvement of women facilitates the spread of ideas within the community, especially about resources such as water systems that women are particularly involved in using and managing daily $(20,21,32,39,60)$.

\section{MARKET}

The market mechanism operates through the dynamic relations between demand and supply which determines the price of a good or service. Price, in turn, determines the extent of demand and quantity of supply of the good or service. The level of demand or supply for a good or service influences the price consumers are willing to pay for it. When a good or service becomes more available (i.e. if supply increases), prices tend to fall, and when the demand for a good or service reduces, prices tend to fall. The same happens if the price of (competing) alternatives to the good or service were to fall. Market failure results when demand and supply are not responsive to each other

\section{Access}

Knowledge mediates relations between supply and demand, lack of knowledge results in imbalances between supply and demand. Without knowledge, access to a resource or service may remain low, even when it is available at an affordable price. Hence, to lead to improved use, efforts to increase and improve the quality of supply (e.g. through training to increase the skills of local artisans on well design), require complementary efforts to increase household knowledge (e.g. through social media and public demonstrations) (68). The greater the level of community dependence on a resource (e.g. high levels of 
need for clean, safe, drinkable water for domestic purposes), the more likely it is that the community will invest in its supply (e.g. by promoting local development of skills required to maintain hardware), thus, helping to maintain or increase access to water $(16,41,43,59,60,69)$.

\section{Sustainability}

Varying levels of demand for a resource or service due to seasonality can have an important influence on sustainability $(48,49)$. For example, water kiosk services are viable during the dry season when people are willing to pay for water because of limited rain and access to water. However, market failure of water kiosks occurs (and they become unviable in the long term) due to continued supply during the wet season in settings where there is increased availability of water due to cheaper options linked to rains then becomes unviable in the long term (51). Willingness to pay for a water system $(17-19,21,51)$ and community-based water supply projects $(52,53)$ often determines a systems reliability or sustainability, and this is linked to demand, affordability and financial capacity in a community. Ability to access resources and funding is important when communities lack knowledge and skills, and the ability to pay for such skills lacking (41).

User fees are often imposed when there is limited internal or external financial support, and the resource or service requires ongoing maintenance and operational costs. User fees can also help to regulate demand and promote responsible use of resources, promoting sustainability $(41,52)$. Low levels of willingness to pay (sometimes reflecting low levels of need or capacity to pay) can become a barrier to operation and maintenance and sustainability of the water service and latrine quality $(16,21,41,52,69)$. Where willingness exists in conjunction with inability to access funds and resources access to water and latrines is compromised $(21,65)$. Willingness to pay may also depend on satisfaction with and quality of the service, which in turn depends on how well it is operated or maintained (19).

\section{OWNERSHIP}

Ownership can be triggered through community engagement, inclusion, empowerment, and sense of achievement. The basis of the mechanism is that the sense of ownership of a resource or hardware leads to feeling committed to it at a community or individual level. People value the resource as they feel invested and want to protect it, thus promoting access to it, and its sustainability. Access and sustainability are the two primary outcomes associated with the ownership mechanism.

\section{Access}


The extent to which community members feel a sense of ownership towards a resource can influence whether they seek external or draw on internal technical and financial support to ensure that the resources (e.g., water and sanitation facilities) are in place $(21,41,46,50,55,70)$. High levels of social cohesion and connectedness is an enabler of efforts to access external funds or mobilise community resources $(21,46,50,70)$. Involvement of a broad range of community members in water and sanitation initiatives can promote a collective sense of ownership, which, in turn, can facilitate social cohesion and connectedness by providing opportunities for a community to share a common goal $(8,71,72)$. By promoting community ownership of a resource, involving community members in capacity building (e.g. in managing the resource) can promote accessibility $(31,32,62)$, and involving women leaders and people with disabilities (e.g., in decision-making) can ensure that access is equitable $(30,38,39,49,71)$.

Communities with higher financial capacity and proximity to latrine products and materials have higher access to latrine (55). The need of the resource can help promote ownership, as the communities have dependency on it and take greater steps to ensure access to resources such as water and latrines $(32,73,74)$. Women communicate with the committees about system breakdowns and without whom, such information sharing that lead to repair may not occur (50). Such active communication reflects ownership and indicate a willingness to work together and build connections within the community which promotes continuous access by facilitating ongoing response to maintenance issues $(32,47,59,63)$.

\section{Sustainability}

Ownership is enabled by involving communities in the design, planning and implementation of a hardware, increasing the likelihood that the resource reflects community needs $(15,72)$, the costs are appropriate and affordable $(16,69)$, the community has the appropriate information for operations and maintenance $(69,75)$, and the community is willing and able to commit to bear hardware costs and ongoing operational and maintenance costs $(16,19,48,76)$, thus becoming self-sufficient $(16,69,70)$. In the absence of this, a community may need to rely on external sources for sustainability of resources $(18,52,75,76)$. Ownership is enabled when communities are allowed or granted decision-making authority in operations and have responsibility for maintenance of hardware (18). Taking ownership requires a leader or committee to champion action $(17,37,41,70)$, especially when a committee has to manage the hardware (e.g. in terms of speed and adequacy of repair), and to raise and manage funds necessary to maintain the hardware (e.g., their water system) in the long term $(39,41,44,59,64,70)$. Financial management ability, can be hampered when communities have low budget resources or communities are unable to afford the service and the committee receives limited funds to maintain a water system, impacting the long-term sustainability of the water service $(15,41)$. 
The capacity to manage the hardware can promote a sense of ownership $(17,21,32,52,72)$. Therefore, retention of human resources and committee members, can lead to sustainability as knowledge and skills are retained in communities $(21,40)$. Having a broad range of members (including women) on such committees can promote buy-in from the community, and diverse voices in decisions leading to a greater sense of ownership, and desire to maintain the resource $(16,25,39,40,50,69)$. Involvement of women is important as they use water regularly and have a great need for water, and in some cases, their sense of ownership can be sufficiently strong to enable them to independently fund, plan, build and maintain water systems (60), allowing them to gain status, take on leadership roles and support younger women in the community $(16,60)$. Lack of ownership of hardware can occur in communities with low socio-economic status as they may have other priorities, or where the need for the hardware is limited, which in turn limits commitment to its maintenance (17).

\section{SHAME}

The shame mechanism generates outcomes due to efforts to avoid feeling disgust or disappointment in one's behaviour or performance. Shame can be leveraged as a feature of interventions which seek to reset community norms, so community members view open defecation and not washing hands as disgusting and as harmful to the whole community. Comparison between individuals, between households and between communities, can lead to shame as it brings out elements of competition and can result in shame from the loss. This may manifest by introducing incentives (e.g. in the form of prizes and rewards) to motivate communities to achieve a water or sanitation goal in comparison to one another $(10,31,33,35,40,62)$. Access and behaviour change are the two outcomes associated with the shame mechanism.

\section{Access}

Without external support, shame can promote access to and individual/household ownership of latrines $(22,31,38,55,57,62,65,77)$ and handwashing facilities $(30,33,62)$. These outcomes are limited in communities and households with financial and technical challenges $(24,35,61,65)$. Enablers include initiating shame by leaders and people known within the community, which helps the acceptance, monitoring and enforcement of new norms $(22,31,55,57,62,77)$. Smaller homogenous communities with effective leadership, are more likely to achieve universal sanitation coverage because people have closer relationships and are more mindful of the impact of their behaviours on others, therefore change in social norms occur more readily (31).

Social cohesion and connectedness are important enablers of household latrine construction because a level of cooperation and collective action it promotes can help overcome the inequality in resources and 
skills necessary to build latrines $(11,23,30,34,46,56,61)$. Together they promote friendship and community respect, and an appreciation of social benefit of private goods that may help reduce open defecation (62). Social cohesion and connectedness also enable 'shame' to be transmitted throughout a community. However, when communities lack technical or financial capabilities $(24,65)$, access may be compromised by construction of low standard latrines, although when the new social norm is sufficiently established, communities may seek to repair or rebuild such latrines $(55,65)$. The shame of losing in a competition can have a negative effect on the non-winners who may be demotivated as a result (62). Improvements in access to latrines and handwashing facilities $(33,62)$ may occur as a result of the motivation of winning prizes (e.g., motorcycles) or simply the right of a leader to claim they 'won' by their community being first to become open defecation free $(31,33)$.

\section{Change}

Efforts to avoid shame can result in change in sanitation behaviour (i.e. reduction in open defecation and increase latrine use $(10,11,24,30,31,34,35,40,65,68,77))$ and an increase in levels of overall hygiene behaviour, including hand washing $(24,25)$ and safe drinking water and storage $(24)$. These changes impact health outcomes such as reduction childhood in diarrheal disease, roundworm infestation and stunting $(31,35,61,65,77)$. Behaviour change is limited where prior beliefs about the negative impact of open defecation are weak (77), where open defecation solves a cultural problem such as men and women not being allowed to share a toilet $(10,11)$, and where there is lack of water for latrine cleaning or hand washing close to latrines (65). Shame is more effective for behaviour change in small, cohesive, and isolated total communities with similar views, as people want to fit in and conform to social norms (68), where effective leadership and community involvement help reinforce change in social norms $(10,30,65)$, and where high social cohesion and connectedness makes people more likely to conform for the benefit of the community $(8,61)$, especially as social pressure mounts and community tolerance for the undesirable behaviour decreases $(11,22,61)$.

Effective leadership is an important enabler of reduced levels of open defecation in communities, as it helps reinforce changes in social norms $(10,30,65)$. High rates of latrine ownership and access, can indicate a chance in social norms and the acceptance of the new behaviour (55). Communities that have a lead role in an intervention, allows them to identify the impacts of their behaviour and helps them understand importance of change in behaviour leading to a paradigm shift to promote sanitation facilities and stop open defecation (65). However, increase in latrine ownership does not always guarantee their sustained use, increase in handwashing or reduction in open defecation $(10,24,55,65)$. Without ongoing support and acknowledgement of change, behaviour change is unlikely to be sustained as there is a tendency for fatigue or loss of motivation within communities (22-24). 


\section{Discussion}

In this realist review, we investigated how (mechanisms) and under what circumstances (context) community efforts and decisions regarding WASH interventions promote health and sustainability (outcomes) in LMICs. To our knowledge, this is the first realist review to do so. We identified five mechanisms: accountability, diffusion, market, ownership, and shame. We also identified contextual factors that lead to positive and negative outcomes for access, behaviour change, health, and sustainability in both external and internal interventions or initiatives in community setting.

Our study identified 19 contextual factors that need to be considered prior to interventions starting including community location, communities with similar characteristics, communication, leadership, fines/penalties, seasonality, resource dependency, access to resources and funding, community financial and technical skills and knowledge, leadership, ongoing support and acknowledgement of change, community connectedness and social cohesion, community willingness to pay, committees with followed and understood responsibilities, rules and management plans, active committees that include women and community/committee involvement in the design, planning or implementation of the intervention. Different combinations of these factors lead to different outcomes. Amongst the mechanisms, overlapping contextual factors were identified including social cohesion and connectedness, skills and knowledge (including financial capabilities and technical abilities to for operations and maintenance), willingness to pay, communication, communities with similar characteristics and views often in a remote location, leadership and diverse involvement in the intervention (of women and at different stages of design, planning and implementation).

Our findings have some similarities with the realist reviews by Loevinsohn et al (5) and Stefanelli et al (7) showing community cohesion and connectedness, community involvement and skills and knowledge as enabling factors. Further, Loevinsohn et al (5) found agencies made the decisions of where and when to intervene, but they had limited transparency and accountability which can lead to inequalities in services and cause the intervention to be different than planned. This reflects the need for key contextual factors we identified to be considered such as leadership and diverse involvement in the intervention. Stefanelli et al's (7) review conducted in four high income countries also found issues with co-management of water resources, the need to redevelop current water governance practices and barriers to implementation such as difficulties engaging Indigenous participants.

In addition, other literature reviews have been conducted to explore contextual factors impacting WASH outcomes and interventions. While Jiménez et al (4) categorised their outcomes differently (legitimacy of the process, increased awareness from the population, participant empowerment, better management and accountability and improved sustainability), their findings resonant with ours. Like our CMO configurations, Jiménez et al developed procedural elements and contextual factors that influence rural drinking water, rural sanitation and urban drinking-water and sanitation. Further, other factors such as access to adequate and timely information were identified (4). What our paper adds, is what factors have positive and negative outcomes. In their systematic review, Novotný et al (6) provide a health-outcome 
nexus assessing how contextual factors impact different sanitation outcomes. Again, similar outcomes and contextual factors were identified, but with a broader range of community and individual socioeconomic factors (6).

Monitoring is a major component of the accountability mechanism, and it can be conducted through multiple forms including reports and follow up visits. However, the downside of ongoing monitoring is that it is expensive, time consuming, labour and resource intensive, and increased frequency can induce reactivity from subjects $(78,79)$. Outsider reporting of activities and changes may also not be accurate (78). The length of time involved in monitoring and observation may be inconvenient for households and communities and may change their routines (79). Higher social economic households show greater responses to monitoring or observation, and may not reflect the population (80).

Diffusion of innovation has been widely identified as a mechanism in public health, for example in the adoption of new health policies and technologies, and use of new drugs (81). In using this mechanism, there is a growing push to highlight the psychological and physical health benefits of what is being adopted (82). New behaviours may need to be adapted to meet the cultural needs of the target population $(81,82)$ prior to adoption. A downside of the diffusion mechanism is that blame can be put on an individual and on those of lower social economic status who are unable to conform or adopt an innovation or new behaviour due to financial barriers (82).

The market mechanism builds on the economic theory of demand and supply. For this mechanism to be sustained there needs to be delicate balance between supply, demand and pricing on the resource (83). The downside of markets is that they may not be sensitive to the cultural and social dimensions involved in the use of water and sanitation services or resources $(84,85)$. Further, the market mechanism, does not take into consideration the effects of climate change and the changing population demands $(84,85)$. Research in the United Kingdom highlighted the difficulty of using the market mechanism to predict underlying patterns to sustain WASH initiatives (84).

Ownership can be broken into several components, including 1) process (who has a voice and whose voice is heard), 2) outcome (who influences the decisions and from what effort), and 3) distribution (who is affected by the process and outcome) (86). Ownership can be difficult to achieve, especially equally and fairly within a community, especially as privilege and socio-economic status is involved in who has a voice, whose voice listened to, who has the power or influence within the community to be involved in decision-making, and who has the power or influence to be involved in negotiations (86). A systematic review found ownership a key component for health intervention sustainability within Sub-Saharan Africa (87).

Shame as a mechanism has been widely used in sanitation, tobacco control and obesity interventions (88-90). Shame has been employed by community-led total sanitation since the 1990s (90). However, there are recent arguments in global health that shame should never be employed as a mechanism to drive improved health outcomes (91), because it can lead to psychological harm especially among poor households who cannot afford to make the change, and because it can be a direct attack on a person's 
identity or concept of self and be detrimental to their self-esteem $(20,92,93)$. The shame mechanism is woven into many behaviour change sanitation interventions. Before using this mechanism, detailed discussions need to occur to highlight potential downsides of focusing on shame.

\section{Strengths And Limitations}

This review involved a thorough detailed search, which identified 56 papers, from 25 countries for inclusion. The size of the review allowed for diverse CMOs to be explored and understood. However, no papers from small island developing states that matched our inclusion criteria were located. Most papers included in the study were from the African and Asian LMICs. Due to this it is unreasonable to generalise the findings to all contexts, we would need to include papers from a wider range of contexts and varied locations. This is an important factor to consider as small island developing states have different priorities and challenges for water and sanitation in comparison to Asian and African countries. Research should be conducted within small island developing states to further refine the $\mathrm{CMO}$ configurations identified in this review and to develop new $\mathrm{CMO}$ configurations which can be used to explain multiple outcomes around water and sanitation interventions in these settings, as the literature have tended to only look at one or the other.

The papers selected for this review were limited to those available in English, peer reviewed and available online through database search. We also only looked at published articles and did not include grey literature such as NGO and government reports. Most of the papers identified only focused on outcomes over a short time period, with only a few looking over five years $(59,69)$. The short time frames are usually insufficient for behaviour change or sustainability to be observed. In the Māori population of New Zealand, a frame of less than 50 years is not appropriate as it does not consider, the 'mokopuna of the mokopuna' [grandchild of the grandchild] (94). Among the papers included in our study it was hard to identify 'physical' and 'social' contextual factors, as very limited information was often provided on these, and details were not deemed relevant information for the paper. If more information was available, then the types of contextual factors may have been found to play a bigger role than we identified. Lastly, we did not include papers that looked at communities' natural resource management and interventions, we only included papers where an external party such as NGO or government was involved in the natural resource management and interventions. Further research needs to be conducted to identify mechanisms involved in such initiatives.

Based on our findings, diverse involvement of communities to assess the design and plan an initiative and intervention is one of the most important enabling factors for sustainability and behaviour change. For water resource focused interventions where the goal is to promote access or sustainability of the resource then the accountability, market and ownership mechanisms seem to be particularly important, but these mechanisms depends more on a broad range of community-level contextual factors. For behaviour change, mechanisms shame and diffusion of innovation seem to be particularly effective, but they are most effective in smaller homogenous communities. Before starting any intervention or initiative, 
it is important to understand the contextual factors within each community, and to tailor the intervention or initiative accordingly.

\section{Conclusion}

This study brings together the knowledge generated from 56 water and sanitation interventions in LMICs where communities are involved. Health, infrastructure and service longevity related outcomes are influenced by five mechanisms. The mechanisms are, 1 ) accountability (policies and procedures to hold communities and committees responsible for their actions and outcomes of an initiative), 2) diffusion (spread of an idea or behaviour by innovators over time through communication amongst members of a community), 3) market (the interplay between demand and supply of a service or resource), 4) ownership (sense of possession and control of the service or resource), and 5) shame (a feeling of disgust in one's behaviour or actions). We identified 19 individual contextual factors that impact these mechanisms, with six overlapping contextual factors. These findings highlight for policy makers, program implementers and NGOs the array of contextual factors that must be considered in the planning phases prior to water and sanitation interventions to improve the likelihood of success for targeted outcomes and infrastructure and service longevity. The results also provide a framework for analysing and understanding the performance of WASH interventions and initiatives retrospectively.

\section{Abbreviations}

LMICs

Low-and-middle income countries

WASH

Water, sanitation and hygiene

\section{Declarations}

Ethics approval and consent to participate

Not applicable

Consent for publication

Not applicable

Availability of data and materials

Data sharing is not applicable to this article as no datasets were generated or analysed during this current study

Competing interests 
The authors declare that they have no competing interests

\section{Funding}

Sarah Nelson is supported by the Indo-Pacific Centre for Health Security, Department of Foreign Affairs and Trade Australia through a PhD scholarship. The funders had no role in the study design, data collection and analysis, decision to publish or preparation of this manuscript

\section{Authors' contributions}

SN was responsible for conceptualization of the study, data extraction, synthesis and interpretation, the literature search and the development of the manuscript. DD was involved in study, data extraction, synthesis and interpretation and the development of the manuscript. AJ and JN were responsible for the data conceptualization and provided feedback on the manuscript. SA was responsible for conceptualization of the study, data synthesis and interpretation, and, development and feedback of the manuscript. All authors read and approved the manuscript

\section{Acknowledgements}

Not applicable

\section{References}

1. Humphrey JH. Reducing the user burden in WASH interventions for low-income countries. Lancet Glob Health. 2019;7(9):e1158-9.

2. Bhutta ZA, Gaffey MF, Crump JA, Steele D, Breiman RF, Mintz ED, et al. Typhoid fever: Way forward. Am J Trop Med Hyg. 2018;99(Suppl 3):89-96.

3. Garn JV, Sclar GD, Freeman MC, Penakalapati G, Alexander KT, Brooks P, et al. The impact of sanitation interventions on latrine coverage and latrine use: a systematic review and meta-analysis. Int J Hyg Environ Health. 2017;220(2):329-40.

4. Jiménez A, LeDeunff H, Giné R, Sjödin J, Cronk R, Murad S, et al. The enabling environment for participation in water and sanitation: a conceptual framework. Water. 2019;11(2):308.

5. Loevinsohn M, Mehta L, Cuming K, Nicol A, Cumming O, Ensink JHJ. The cost of a knowledge silo: A systematic re-review of water, sanitation and hygiene interventions. Health Policy Plan. 2015;30(5):660-74.

6. Novotný J, Hasman J, Lepič M. Contextual factors and motivations affecting rural community sanitation in low- and middle-income countries: a systematic review. Int J Hyg Environ Health. 2018;221(2):121-33.

7. Stefanelli RD, Castleden H, Harper SL, Martin D, Cunsolo A, Hart C. Experiences with integrative indigenous and western knowledge in water research and management: a systematic realist review 
of literature from Canada, Australia, New Zealand, and the United States. Environ Rev. 2017;25(3):323-33.

8. Harter M, Mosch S, Mosler H-J. How does community-led total sanitation (CLTS) affect latrine ownership? A quantitative case study from Mozambique. BMC Public Health. 2018;18(1):387.

9. Valcourt N, Walters J, Javernick-Will A, Linden K, Hailegiorgis B. Understanding rural water services as a complex system: an assessment of key factors as potential leverage points for improved service sustainability. Sustainability. 2020;12(3):1243.

10. Zimba R, Ngulube V, Lukama C, Manangi A, Tiwari A, Osbert N, et al. Chiengi District, Zambia open defecation free after 1 year of community-led total sanitation. Am J Trop Med Hyg. 2016;95(4):9257.

11. Mlenga DH, Baraki YA. Community led total sanitation for community based disaster risk reduction: a case for non-input humanitarian relief. Jàmbá J Disaster Risk Stud. 2016;8(2).

12. Jennewein JS, Jones KW. Examining 'willingness to participate'in community-based water resource management in a transboundary conservation area in Central America. Water Policy. 2016;18(6):1334-52.

13. McGinnis MD, Ostrom E. Social-ecological system framework: Initial changes and continuing challenges. Ecol Soc. 2014;19(2).

14. Foster T. Predictors of sustainability for community-managed handpumps in Sub-Saharan Africa: evidence from Liberia, Sierra Leone, and Uganda. Environ Sci Technol. 2013;47(21):12037-46.

15. Abedin MdA, Habiba U, Shaw R. Community perception and adaptation to safe drinking water scarcity: salinity, arsenic, and drought risks in coastal Bangladesh. Int J Disaster Risk Sci. 2014;5(2):110-24.

16. Padawangi R. Community-driven development as a driver of change: water supply and sanitation projects in rural Punjab, Pakistan. Water Policy Oxf. 2010;12(S1):104-20.

17. Klug T, Shields KF, Cronk R, Kelly E, Behnke N, Lee K, et al. Water system hardware and management rehabilitation: qualitative evidence from Ghana, Kenya, and Zambia. Int J Hyg Environ Health. 2017;220(3):531-8.

18. Jimenez-Redal R, Soriano J, Holowko N, Almandoz J, Arregui F. Assessing sustainability of rural gravity-fed water schemes on Idjwi Island, D.R. Congo. Int J Water Resour Dev. 2018;34(6):1022-35.

19. Kwangware J, Mayo A, Hoko Z. Sustainability of donor-funded rural water supply and sanitation projects in Mbire district. Zimbabwe Phys Chem Earth. 2014;76-78:134-9.

20. Jones S. Participation as citizenship or payment? A case study of rural drinking water governance in Mali. 2011;4(1):18.

21. Madrigal-Ballestero R, Naranjo MA. Adaptive capacity, drought and the performance of communitybased drinking water organizations in Costa Rica. J Water Clim Change. 2015;6(4):831-47.

22. Russpatrick S, Tiwari A, Markle L, Musonda E, Mutunda A, Osbert N, et al. Mobility up the sanitation ladder following community-led total sanitation in rural Zambia. J Water Sanit Hyg Dev. 
2017;7(3):436-44.

23. Ogendo KN, Kihara AB, Kosgei RJ, Tweya H, Kizito W, Murkomen B, et al. Assessment of community led total sanitation uptake in rural Kenya. East Afr Med J. 2016;5.

24. Woode PK, Dwumfour-Asare B, Nyarko KB, Appiah-Effah E. Cost and effectiveness of water, sanitation and hygiene promotion intervention in Ghana: the case of four communities in the Brong Ahafo region. Heliyon. 2018;4(10).

25. Madon S, Malecela MN, Mashoto K, Donohue R, Mubyazi G, Michael E. The role of community participation for sustainable integrated neglected tropical diseases and water, sanitation and hygiene intervention programs: a pilot project in Tanzania. Soc Sci Med. 2018;202:28-37.

26. Rasanathan K, Bennett S, Atkins V, Beschel R, Carrasquilla G, Charles J, et al. Governing multisectoral action for health in low- and middle-income countries. PLoS Med. 2017;14(4).

27. Naiga R, Penker M. Determinants of users' willingness to contribute to safe water provision in rural Uganda. Lex Localis. 2014;12(3):695.

28. Pawson R, Greenhalgh T, Harvey G, Walshe K. Realist review - a new method of systematic review designed for complex policy interventions. J Health Serv Res Policy. 2005;10(Suppl 1):21-34.

29. Wong G, Greenhalgh T, Westhorp G, Buckingham J, Pawson R. RAMESES publication standards: realist syntheses. BMC Med. 2013;11(1):21.

30. Crocker J, Geremew A, Atalie F, Yetie M, Bartram J. Teachers and sanitation promotion: an assessment of community-led total sanitation in Ethiopia. Environ Sci Technol. 2016;50(12):651725.

31. Tiwari A, Russpatrick S, Hoehne A, Matimelo SM, Mazimba S, Nkhata I, et al. Assessing the impact of leveraging traditional leadership on access to sanitation in rural Zambia. Am J Trop Med Hyg. 2017;97(5):1355-61.

32. Maheshwari B, Varua M, Ward J, Packham R, Chinnasamy P, Dashora Y, et al. The role of transdisciplinary approach and community participation in village scale groundwater management: insights from Gujarat and Rajasthan, India. Water. 2014;6(11):3386-408.

33. Mwakitalima A, Massa K, Seleman A, Kassile T. Scaling up rural sanitation in Tanzania: evidence from the national sanitation campaign. J Water Sanit Hyg Dev Lond. 2018;8(2):290-306.

34. Crocker J, Abodoo E, Asamani D, Domapielle W, Gyapong B, Bartram J. Impact evaluation of training natural leaders during a community-led total sanitation intervention: A cluster-randomized field trial in Ghana. Environ Sci Technol. 2016;50(16):8867-75.

35. Pickering AJ, Djebbari H, Lopez C, Coulibaly M, Alzua ML. Effect of a community-led sanitation intervention on child diarrhoea and child growth in rural Mali: a cluster-randomised controlled trial. Lancet Glob Health. 2015;3(11):e701-11.

36. World Bank. World Bank Country and Lending Groups [Internet]. [cited 2019 May 5]. Available from: .

37. Lodenstein E, Dieleman M, Gerretsen B, Broerse JEW. Health provider responsiveness to social accountability initiatives in low- and middle-income countries: a realist review. Health Policy Plan. 
2017;32(1):125-40.

38. Biran A, Danquah L, Chunga J, Schmidt W-P, Holm R, Itimu-Phiri A, et al. A cluster-randomized trial to evaluate the impact of an inclusive, community-led total sanitation intervention on sanitation access for people with disabilities in Malawi. Am J Trop Med Hyg. 2018;98(4):984-94.

39. Anthonj C, Fleming L, Cronk R, Godfrey S, Ambelu A, Bevan J, et al. Improving monitoring and water point functionality in rural Ethiopia. Water. 2018;10(11):1591.

40. Kema KM, Komwihangiro J, Kimaro S. Integrated community based child survival, reproductive health and water and sanitation program in Mkuranga district, Tanzania: a replicable model of good practices in community based health care. Pan Afr Med J. 2012;13(Suppl 1).

41. Roekmi RAK, Baskaran K, Chua LH. Community-based water supplies in Cikarang, Indonesia: Are they sustainable? Nat Resour Forum. 2018;42(2):108-22.

42. Gimaiyo G, McManus J, Yarri M, Singh S, Trevett A, Moloney G, et al. Can child-focused sanitation and nutrition programming improve health practices and outcomes? Evidence from a randomised controlled trial in Kitui County, Kenya. BMJ Glob Health. 2019;4(1).

43. Sarkar S, Greenleaf JE, Gupta A, Ghosh D, Blaney LM, Bandyopadhyay P, et al. Evolution of community-based arsenic removal systems in remote villages in West Bengal, India: assessment of decade-long operation. Water Res. 2010;44(19):5813-22.

44. Madziyauswa V. Assessing sustainability of community managed NGOs' WASH interventions in rural Zimbabwe: the case of Chivi district in Masvingo province. J Water Sanit Hyg Dev. 2017;640-9.

45. Keoprasith B, Kizuki M, Watanabe M, Takano T. The impact of community-based, workshop activities in multiple local dialects on the vaccination coverage, sanitary living and the health status of multiethnic populations in Lao PDR. Health Promot Int. 2013;28(3):453-65.

46. Dickin S, Bisung E, Savadogo K. Sanitation and the commons: the role of collective action in sanitation use. Geoforum. 2017;86:118-26.

47. Madrigal-Ballestero R, Naranjo MA. Adaptive capacity, drought and the performance of communitybased drinking water organizations in Costa Rica. J Water Clim Change. 2015;6(4):831-47.

48. Opare S. Sustaining water supply through a phased community management approach: lessons from Ghana's “oats” water supply scheme. Environ Dev Sustain. 2011;13(6):1021-42.

49. Morinville C, Harris LM. Participation, politics, and panaceas: exploring the possibilities and limits of participatory urban water governance in Accra, Ghana. Ecol Soc. 2014;19(3):art36.

50. Kelly E, Lee K, Shields KF, Cronk R, Behnke N, Klug T, et al. The role of social capital and sense of ownership in rural community-managed water systems: qualitative evidence from Ghana, Kenya, and Zambia. J Rural Stud. 2017;56:156-66.

51. Kelly E, Shields KF, Cronk R, Lee K, Behnke N, Klug T, et al. Seasonality, water use and community management of water systems in rural settings: qualitative evidence from Ghana, Kenya, and Zambia. Sci Total Environ. 2018;628-629:715-21. 
52. Rout S. Institutional variations in practice of demand responsive approach: evidence from rural water supply in India. Water Policy. 2014;16(4):650-68.

53. Ibrahim SH. Sustainability assessment and identification of determinants in community-based water supply projects using partial least squares path model. J Sustain Dev Energy Water Environ Syst. 2017;5(3):345-58.

54. Rogers EM. Diffusion of Innovations, 5th Edition. Simon and Schuster; 2010.

55. Crocker J, Saywell D, Bartram J. Sustainability of community-led total sanitation outcomes: evidence from Ethiopia and Ghana. Int J Hyg Environ Health. 2017;220(3):551-7.

56. Nunbogu AM, Harter M, Mosler H-J. Factors associated with levels of latrine completion and consequent latrine use in Northern Ghana. Int J Environ Res Public Health. 2019;16(6):920.

57. Zeleke DA, Gelaye KA, Mekonnen FA. Community-led total sanitation and the rate of latrine ownership. BMC Res Notes. 2019;12(1):14.

58. Andrade EL, Bingenheimer JB, Edberg MC, Zoerhoff KL, Putzer EM. Evaluating the effectiveness of a community-based hygiene promotion program in a rural Salvadoran setting. Glob Health Promot StDenis Cedex. 2019;26(1):69-80.

59. Wardle C, Zakiriaeva N. Sustainability and long-term impact of community-managed water supply in rural Kyrgyzstan, Central Asia. Waterlines. 2018;37(2):118-31.

60. Aladuwaka S, Momsen J. Sustainable development, water resources management and women's empowerment: the wanaraniya water project in Sri Lanka. Gend Dev. 2010;18(1):43-58.

61. Cameron L, Olivia S, Shah M. Scaling up sanitation: evidence from an RCT in Indonesia. J Dev Econ. 2019;138:1-16.

62. Whaley $\mathrm{L}$, Webster $\mathrm{J}$. The effectiveness and sustainability of two demand-driven sanitation and hygiene approaches in Zimbabwe. J Water Sanit Hyg Dev. 2011;1(1):20-36.

63. Chankova S, Hatt LE, Musange SF. A community-based approach to promote household water treatment in Rwanda. J Water Health. 2012;10(1):116-29.

64. Singh C. Is participatory watershed development building local adaptive capacity? Findings from a case study in Rajasthan, India. Environ Dev. 2018;25:43-58.

65. Tessema RA. Assessment of the implementation of community-led total sanitation, hygiene, and associated factors in Diretiyara district, Eastern Ethiopia. PLOS ONE. 2017;12(4):e0175233.

66. Ganing A, Abu A, Harpenas, Muslimin I, Adam A. Community empowerment in management community-based total sanitation through health education in Majene. Indian J Public Health Res Dev. 2018;9(12):1466-71.

67. Barrington D, Fuller K, McMillan A. Water safety planning: adapting the existing approach to community-managed systems in rural Nepal. J Water Sanit Hyg Dev. 2013;3(3):392-401.

68. Harvey PA. Zero subsidy strategies for accelerating access to rural water and sanitation services. Water Sci Technol Lond. 2011;63(5):1037-43. 
69. Kwangware J, Mayo A, Hoko Z. Sustainability of donor-funded rural water supply and sanitation projects in Mbire district. Zimbabwe Phys Chem Earth Parts ABC. 2014;76-78:134-9.

70. Behnke NL, Klug T, Cronk R, Shields KF, Lee K, Kelly ER, et al. Resource mobilization for communitymanaged rural water systems: evidence from Ghana, Kenya, and Zambia. J Clean Prod. 2017;156:437-44.

71. Kosinski KC, Crocker JJ, Durant JL, Osabutey D, Adjei MN, Gute DM. A novel community-based water recreation area for schistosomiasis control in rural Ghana. J Water Sanit Hyg Dev Lond. 2011;1(4):259-68.

72. Hubbard B, Sarisky J, Gelting R, Baffigo V, Seminario R, Centurion C. A community demand-driven approach toward sustainable water and sanitation infrastructure development. Int J Hyg Environ Health. 2011;214(4):326-34.

73. Tiwari A, Russpatrick S, Hoehne A, Matimelo SM, Mazimba S, Nkhata I, et al. Assessing the Impact of Leveraging Traditional Leadership on Access to Sanitation in Rural Zambia. Am J Trop Med Hyg. 2017 Nov;97(5):1355-61.

74. Whaley L, Webster J. The effectiveness and sustainability of two demand-driven sanitation and hygiene approaches in Zimbabwe. J Water Sanit Hyg Dev. 2011 Mar;1(1):20-36.

75. Smyrilli C, Selvakumaran S, Alderson M, Pizarro A, Almendrades D, Harris B, et al. Sustainable decentralised wastewater treatment schemes in the context of Lobitos, Peru. J Environ Eng Sci. 2018;13(1):8-16.

76. Bright-Davies L, Lüthi C, Jachnow A. DEWATS for urban Nepal: a comparative assessment for community wastewater management. Waterlines. 2015;34(2):119-38.

77. Degebasa MZ, Weldemichael DZ, Marama MT. Diarrheal status and associated factors in under five years old children in relation to implemented and unimplemented community-led total sanitation and hygiene in Yaya Gulele in 2017. Pediatr Health Med Ther. 2018;9:109-21.

78. Thomas E, Andrés LA, Borja-Vega C, Sturzenegger G. Innovations in WASH Impact Measures: Water and Sanitation Measurement Technologies and Practices to Inform the Sustainable Development Goals. World Bank Publications; 2018. 131 p.

79. Halder AK, Molyneaux JW, Luby SP, Ram PK. Impact of duration of structured observations on measurement of handwashing behavior at critical times. BMC Public Health. 2013;13(1):705.

80. Ram PK, Halder AK, Granger SP, Jones T, Hall P, Hitchcock D, et al. Is structured observation a valid technique to measure handwashing behavior? Use of acceleration sensors embedded in soap to assess reactivity to structured observation. Am J Trop Med Hyg. 2010;83(5):1070-6.

81. Green LW, Ottoson JM, García C, Hiatt RA. Diffusion theory and knowledge dissemination, utilization, and integration in public health. Annu Rev Public Health. 2009;30(1):151-74.

82. Haider M, Kreps GL. Forty years of diffusion of innovations: utility and value in public health. J Health Commun. 2004;9(Suppl 1):3-11.

83. Hicks JR. A Revision of Demand Theory [Internet]. Oxford University Press; 1986 [cited 2019 Nov 26]. Available from: . 
84. Parker JM, Wilby RL. Quantifying household water demand: a review of theory and practice in the UK. Water Resour Manag. 2013;27(4):981-1011.

85. Johansson RC, Tsur Y, Roe TL, Doukkali R, Dinar A. Pricing irrigation water: a review of theory and practice. Water Policy. 2002;4(2):173-99.

86. Lachapelle P. A sense of ownership in community development: understanding the potential for participation in community planning efforts. Community Dev. 2008;39(2):52-9.

87. Iwelunmor J, Blackstone S, Veira D, Nwaozuru U, Airhihenbuwa C, Munodawafa D, et al. Toward the sustainability of health interventions implemented in sub-Saharan Africa: a systematic review and conceptual framework. Implement Sci IS. 2016;11.

88. Riley KE, Ulrich MR, Hamann HA, Ostroff JS. Decreasing smoking but increasing stigma? Antitobacco campaigns, public health, and cancer care. AMA J Ethics. 2017;19(5):475-85.

89. Puhl RM, Heuer CA. Obesity stigma: important considerations for public health. Am J Public Health. 2010;100(6):1019-28.

90. UNICEF

UNICEF. Field Notes: UNICEF Policy and Programming in Practice [Internet]. 2009. Available from: .

91. Brewis A, Wutich A. Why we should never do it: stigma as a behaviour change tool in global health. BMJ Glob Health. 2019;4(5):e001911.

92. Lewis H. Shame and guilt in neurosis. Psychoanal Rev. 1971;58(3):419-38.

93. Bateman M, Engel S. To shame or not to shame-that is the sanitation question. Dev Policy Rev. 2018;36(2):155-73.

94. Morgan TKKB. An indigenous perspective on water recycling. Desalination. 2006;187(1-3):127-36.

\section{Table 1}

Due to technical limitations, table 1 is only available as a download in the supplemental files section.

\section{Figures}


Figure 1: Results of literature search

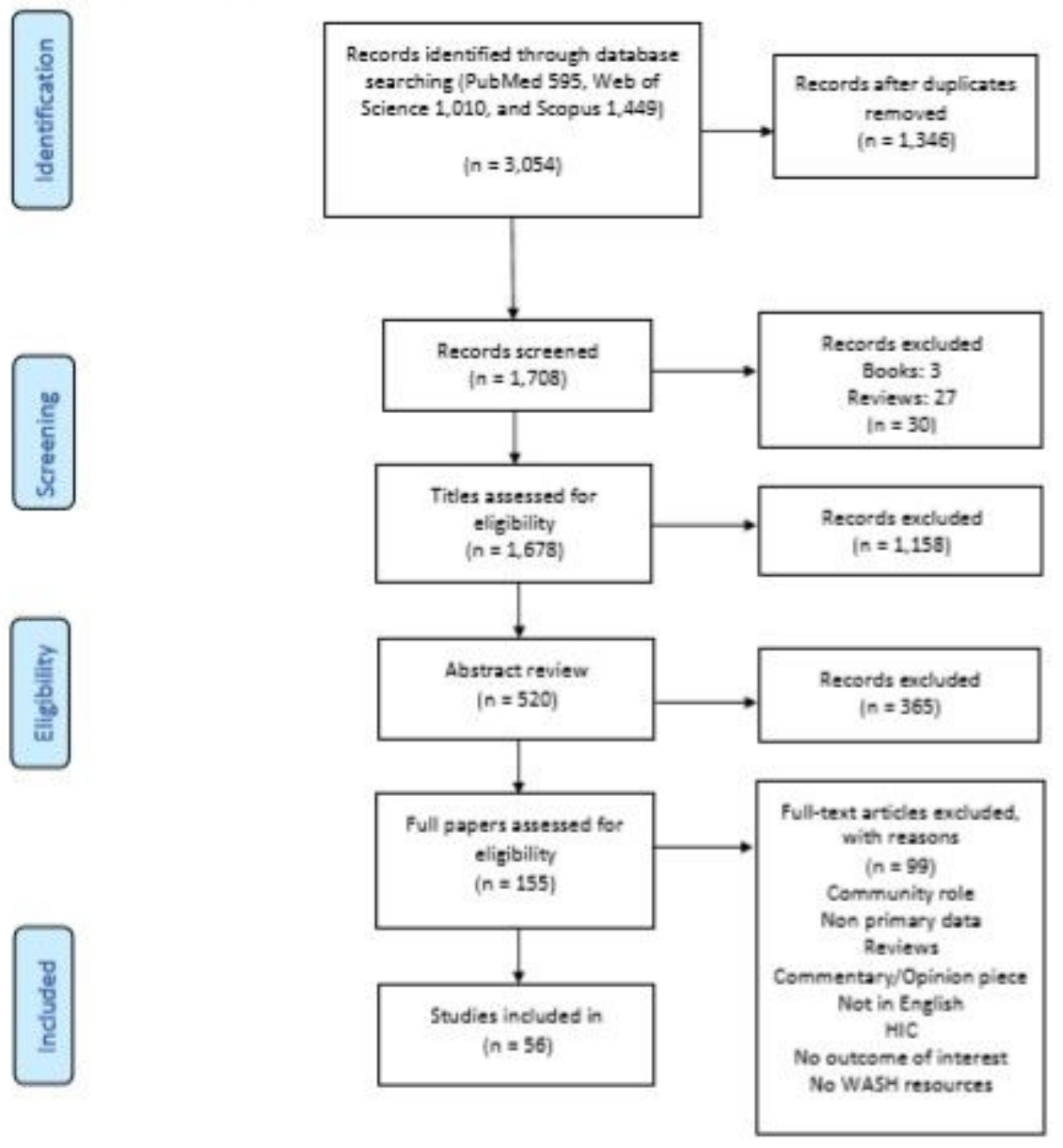

\section{Figure 1}

Results of literature search

\section{Supplementary Files}

This is a list of supplementary files associated with this preprint. Click to download.

- Table1EnablingCMOs.pdf 